\title{
Lyme borreliosis, ticks and Borrelia species
}

\author{
Gerold Stanek
}

(C) Springer-Verlag GmbH Austria, part of Springer Nature 2018

This issue of the Wiener klinische Wochenschrift contains review papers on clinical and diagnostic aspects of Lyme borreliosis, on hard ticks as vectors of human pathogens and on recent discussion on the taxonomy of borreliae. We will learn about approaches for the management of infectious diseases transmitted by hard ticks established during the past 30 years at the Lyme Disease Diagnostic Center, New York Medical College, Valhalla, NY, USA [1]. We also include an article on Viennese long-term experience with Borrelia burgdorferi sensu lato infections that has enabled the diagnosis and successful treatment of rare manifestations of Lyme neuroborreliosis presenting as dementia-like syndromes [2]. An article on ticks as vectors explains what makes ixodid ticks such efficient transmitters of pathogens [3]. In addition, a paper on the proposal for a new genus name for the Borrelia burgdorferi sensu lato complex, which has triggered fierce debate, illuminates the background from the viewpoint of a molecular microbiologist [4].

The terms Lyme borreliosis or Lyme disease have also become terms for medically unexplained symptoms such as fatigue, joint pain, muscle pain and difficulties with memory and concentration [5]. It is useful, therefore, to outline here what Lyme borreliosis includes.

Lyme borreliosis is caused by certain genospecies of the genus Borrelia. These are bacteria of the spirochete family, highly motile through possession of endoflagella. Lyme borreliae are host-dependent bacteria and cannot survive outside a host; however, cultiva-

G. Stanek, M.D. ( $₫)$

Center for Pathophysiology, Infectiology and Immunology, Institute for Hygiene and Applied Immunology, Medical University of Vienna, Kinderspitalgasse 15, 1090 Vienna, Austria

gerold.stanek@meduniwien.ac.at tion is possible under microaerophilic conditions in complex culture media. Reservoir-competent animals include small mammals and certain species of birds (Figs. 1 and 2). The vectors/carriers are ticks of the genus Ixodes (hard ticks) in temperate climates of the northern hemisphere: I. ricinus in Europe, I. persulcatus in north-eastern Europe and Asia, I. scapularis in north-east and mid-west USA; and I. pacificus in western USA.

The infection cycle is linked to the blood meals of the respective development stages of the ticks: larvae, nymphs and adults. About $30 \%$ of nymphal ticks in central Europe are infected with Lyme borreliae. The risk of infection for humans is highest from May to September. Lyme borreliae were discovered in 1982 and identified in 1984 as a new Borrelia species, named Borrelia burgdorferi in honour of its discoverer Willy Burgdorfer. More than 20 genospecies are now included under the umbrella term B. burgdorferi sensu lato. In Europe, the species pathogenic for humans are B. afzelii, B. garinii, B. bavariensis, B. burgdorferi sensu stricto, and occasionally B. spielmanii and B. lusitaniae; in North America, B. burgdorferi sensu stricto and in certain areas B. mayonii. The transmission of Lyme borreliae occurs on average $24 \mathrm{~h}$ $(6-48 \mathrm{~h})$ after a blood meal. There is no transmission from person to person and there is no evidence of diaplacental transmission.

Clinical manifestations include the following:

Erythema migrans An enlarging red or bluish-red spot with or without central clearing, periphery clearly distinct, often more intensely colored, not noticeably elevated, $\geq 5 \mathrm{~cm}$ in diameter; if less, then requirements for diagnosis are: tick bite in medical history, at least 2 days delayed occurrence of erythema after tick bite, and expanding erythema at the site of the bite. Concomitant symptoms: local itching/burning in 38\%, fa- 


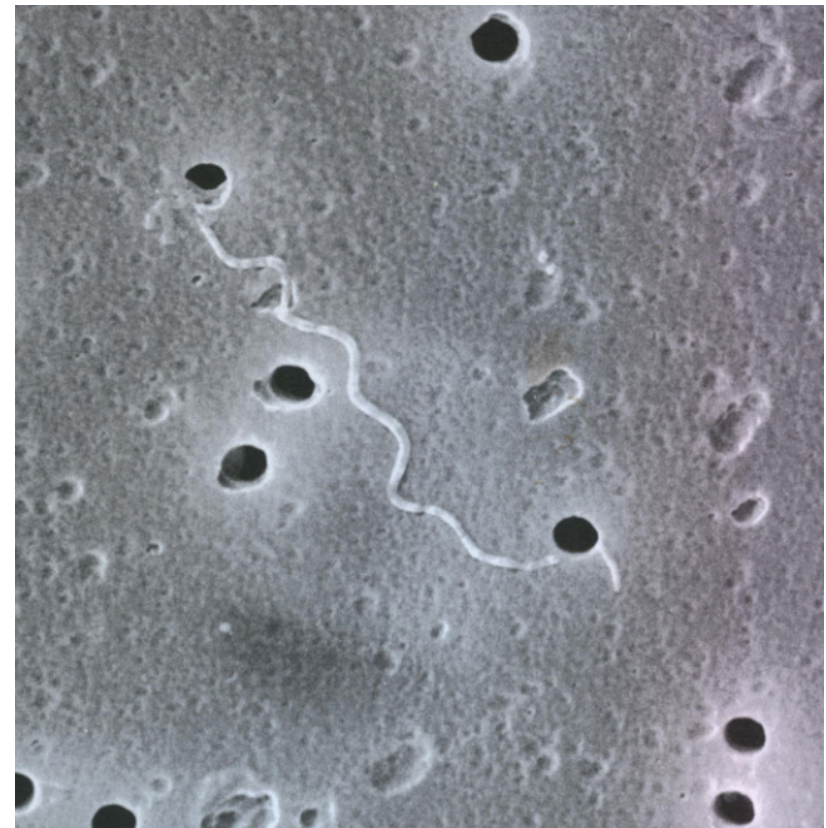

Fig. 1 Borrelia garinii, scanning electron microscopy; pore diameter $1 \mu \mathrm{m}$; diameter of spirochete $0.25 \mu \mathrm{m}$, length ca $20 \mu \mathrm{m}$ (Photo by Phillip Szkokan)

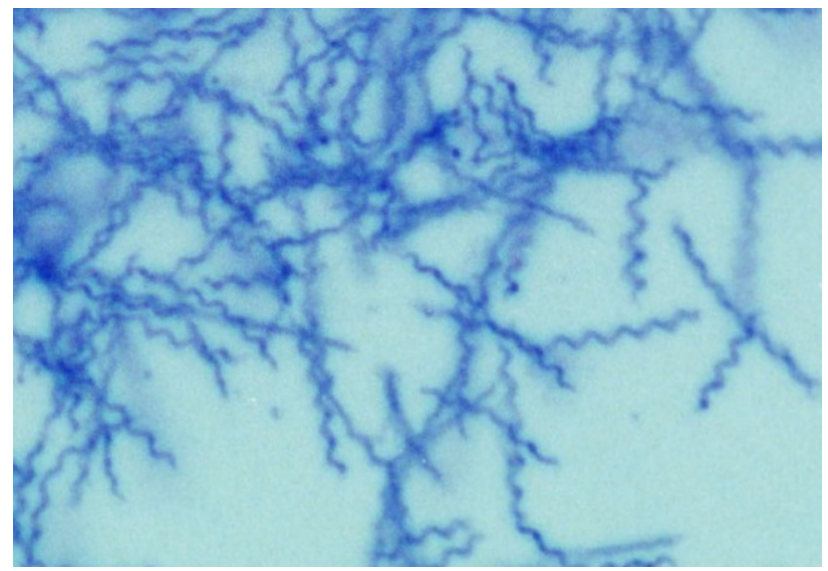

Fig. 2 Borrelia afzelii in culture medium; dark field microscopy, reverse colour image (Photo by Gerold Stanek)

tigue $19 \%$, headache $14 \%$, arthralgia $11 \%$, myalgia $6 \%$ (Figs. 3 and 4).

Borrelial lymphocytoma Painless, bluish red nodule or spot, usually on the earlobe, ear helix, nipple or scrotum; more common in children (especially at the ear) than in adults.

Acrodermatitis chronica atrophicans Long standing red or blue-red lesions, usually on the extensor surfaces of the extremities. The lesions later become atrophic. Thickening of the skin and fibroid nodules on bony prominences may occur (Fig. 5).

Neurologic manifestations Neurologic manifestations are summarized under the term Lyme neu-

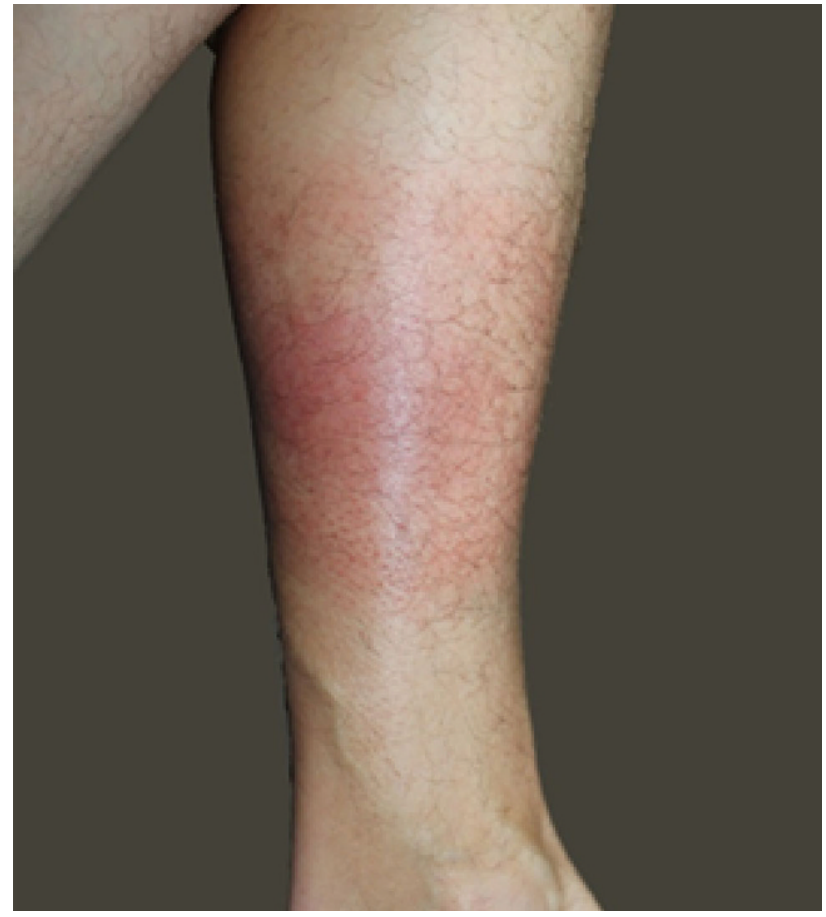

Fig. 3 Erythema migrans left lower leg, tick bite not recognized (Photo by Mateusz Markowicz)

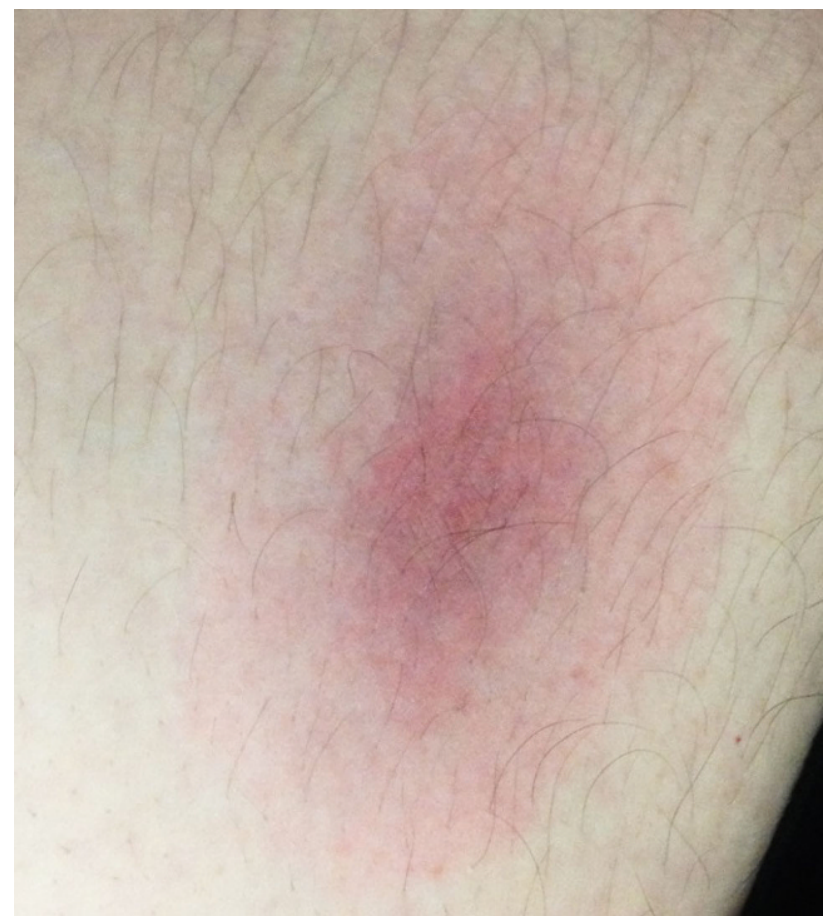

Fig. 4 Erythema migrans left thigh, 10 days after tick bite (Photo by Mateusz Markowicz)

roborreliosis. In adults mainly meningopolyradiculitis (Bannwarth's syndrome), meningitis, rare encephalitis, myelitis, and very rarely cerebral vasculitis. Children present mainly with facial palsy and/or mild meningitis. 


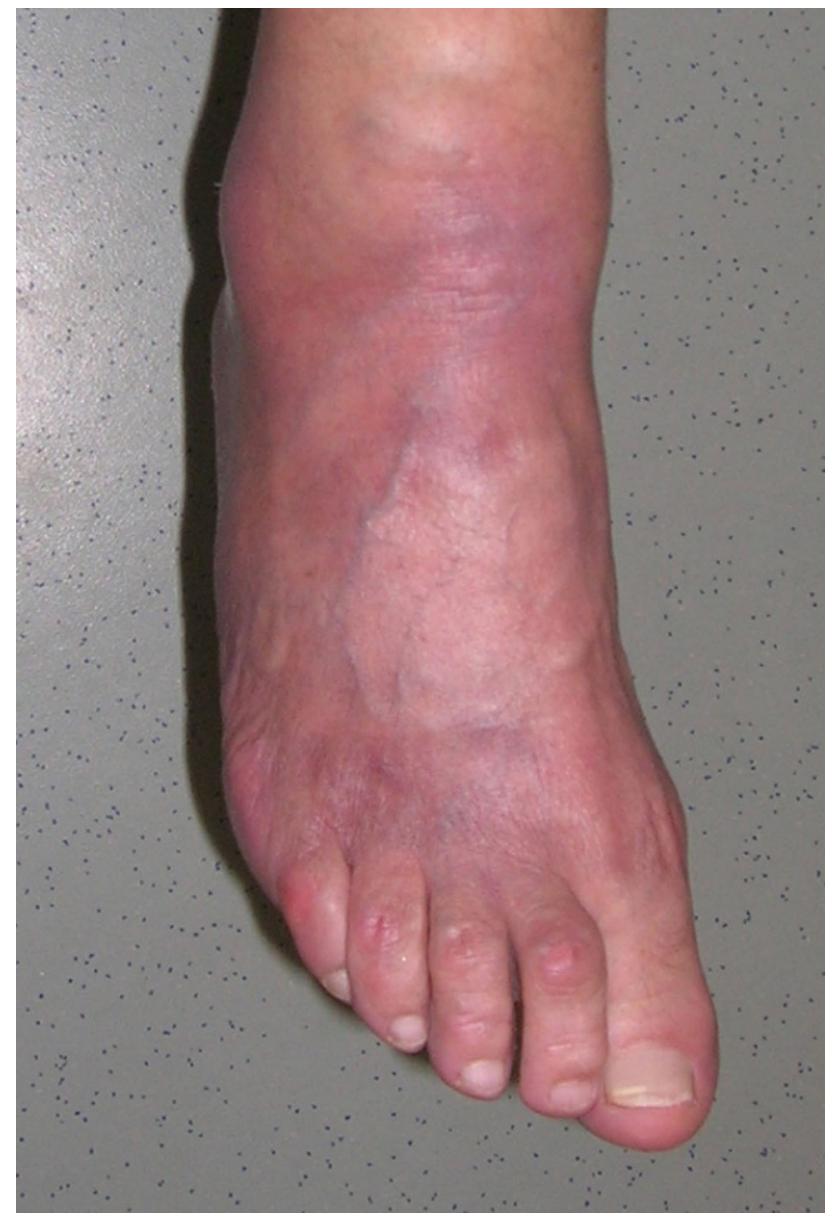

Fig. 5 Acrodermatitis chronica atrophicans right foot (Photo by Mateusz Markowicz)

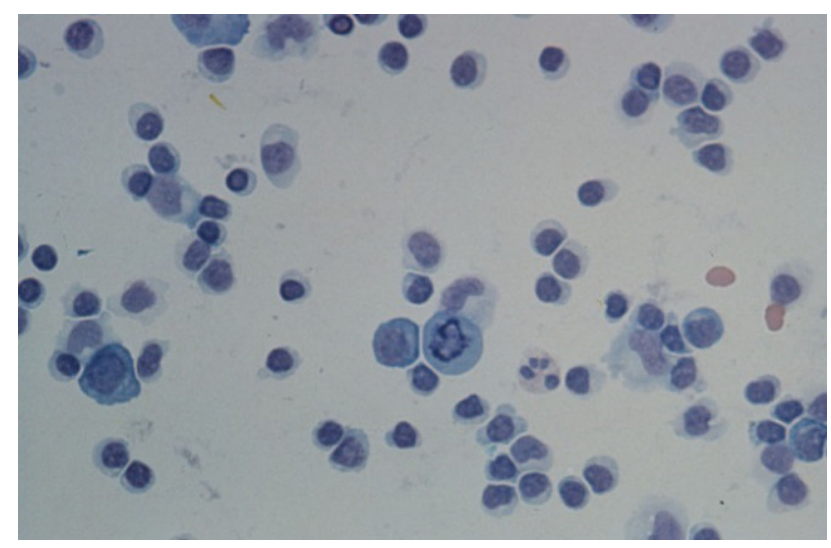

Fig. 6 Cerebrospinal fluid: lymphoplasmacelluar pleocytosis in a case of meningopolyneuritis, May-Grünwald-Giemsa staining. (Courtesy of Wolfgang Kristoferitsch)

Lyme arthritis Recurrent attacks or persistent, objective joint swelling in one or a few large joints. Exclusion of other causes necessary.

Lyme carditis Rare: acute atrioventricular (I-III) conduction disorder, arrhythmia; also possible my- ocarditis, pancarditis. Exclusion of other causes necessary.

Eye manifestations Very rare: conjunctivitis, uveitis, papillitis, episcleritis, keratitis.

Microbiological diagnostics:

Suitable specimens are full blood, EDTA blood and citrate blood; cerebrospinal fluid, synovia, synovialis, and biopsies, primarily skin biopsy.

Direct detection of the pathogens Direct detection of borreliae by cultivation and/or nucleic amplification techniques (PCR). In controlled clinical trials, these techniques for detection of borreliae in body fluids, tissue samples, biopsies, and ticks should be limited to specialized laboratories.

Indirect detection of borrelial infection Detection of specific antibodies should be requested only when there is sound clinical suspicion and according to diagnostic/management guidelines [6]. Results of untargeted searches have very poor predictive diagnostic value. Principle of serology: a two-tier procedure. The first tier using immunofluorescence assay, enzymelinked immunosorbent assay, chemiluminescence, etc. detects antibodies of classes IgG and IgM against $B$. burgdorferi sensu lato; the second tier uses immunoblotting, line-blotting or other technique with a relevant number of immunodominant Borrelia antigens to verify specificity. Seropositivity requires that both the first and the second tier are positive. Serological methods have become established as a diagnostic aid in suspected Lyme borreliosis and numerous products from different manufacturers are on the market. The principle of the two-tier procedure still pertains. The band pattern of an immunoblot together with the relative concentration of antibodies in the first tier screening test allows greater certainty in the diagnosis in clinical manifestations with a broad spectrum of differential diagnoses, such as arthritis. Nonetheless, a positive blot pattern does not indicate a definite clinical manifestation of Lyme borreliosis or an active event. Without clinical signs and symptoms there is no disease.

The best diagnostic aid provides detection of intrathecally produced specific antibodies in the cerebrospinal fluid (CSF), recorded as the CSF/serum antibody index. Together with supportive clinical symptoms and inflammatory CSF (lymphoplasmacellular pleocytosis) findings, the clinically suspected diagnosis is confirmed (Fig. 6).

A persisting delusion is that the mere serological result "Borrelia IgM positive" requires antibiotic treatment. It should be remembered that the prevalence of serum antibodies to B. burgdorferi sensu lato in healthy adults is, e.g. in Austria, approximately $23 \%$ (20-30\%) for IgG and about 18\% (14-23\%) for IgM.

In the following serological constellations, and in the absence of signs and symptoms of Lyme borre- 
liosis, antibiotic treatment should be carefully considered: a) IgM and/or IgG positive without signs of disease $\rightarrow$ no treatment. Laboratories should refrain from interpreting as "active Lyme borreliosis, antibiotic treatment required"; b) IgM and IgG negative in a patient with erythema migrans $\rightarrow$ treatment is necessary (comment: erythema migrans is a clinical diagnosis, no reason for serology); c) IgM negative, IgG positive without signs of disease: in most cases this indicates an earlier Borrelia spp. infection (borrelial IgG antibodies can persist for long periods of time) and antibiotic treatment is certainly not indicated.

Treatment with antibiotics is usually for 2-4 weeks and most patients recover uneventfully. Detailed information on the manifestations, diagnosis and treatment of Lyme borreliosis may be obtained from the references listed below $[7,8]$.

The publications in this issue of the Wiener klinische Wochenschrift should be considered a stimulus to obtain further information on Lyme borreliosis, by review and original work, and on other tick-borne diseases.

Conflict of interest G. Stanek is an unpaid member of the steering committee of ESGBOR, the European Society of Clinical Microbiology and Infectious Diseases (ESCMID) Study Group on Lyme Borreliosis.

\section{References}

1. Wormser GP, McKenna D, Nowakowski I. Management approaches for suspected and established Lyme disease used at the Lyme disease diagnostic center. Wien Klin Wochenschr. 2016; https://doi.org/10.1007/s00508-0150936-y.

2. Kristoferitsch $W$ et al. Secondary dementia due to Lyme neuroborreliosis. Wien Klin Wochenschr. https://doi.org/ 10.1007/s00508-018-1361-9.

3. Kahl O. Hard ticks as vectors-some basic issues. Wien Klin Wochenschr. https://doi.org/10.1007/s00508-018-1360-x.

4. Bergström S, Normark J. Microbiological features distinguishing Lyme disease and relapsing fever spirochetes. Wien Klin Wochenschr. https://doi.org/10.1007/s00508018-1368-2.

5. Wormser GP. Should patients infected with Borrelia burgdorferi nolonger be referred to as having Lyme disease? Am J Med. 2018; https://doi.org/10.1016/j.amjmed.2018. 06.014 .

6. Stanek G, Fingerle V, Hunfeld KP, Jaulhac B, Kaiser R, Krause A, Kristoferitsch W, O'Connell S, Ornstein K, Strle F, Gray J. Lyme borreliosis: clinical case definitions for diagnosis and management in Europe. Clin Microbiol Infect. 2011;17:69-79. https://doi.org/10.1111/j.14690691.2010.03175.x.

7. Stanek G, Wormser GP, Gray J, Strle F. Lyme borreliosis. Lancet. 2012;379:461-73. https://doi.org/10.1016/S01406736(11)60103-7.

8. Stanek G, Strle F. Lyme borreliosis-from tick bite to diagnosis and treatment. FEMS Microbiol Rev. 2018;42:233-58. https://doi.org/10.1093/femsre/fux047. 